\title{
Face Identification through Learned Image High Feature Video Frame Works
}

\author{
Boya Akhila $^{1 *}$, Burgubai Jyothi ${ }^{2}$ \\ ${ }^{1}$ Department of ECE,MRCET, India \\ ${ }^{2}$ Department of ECE,MRCET, India
}

Available online at: www.isroset.org

Accepted: 27/Jun/2018, Online: 31/Aug/2018

\begin{abstract}
The affluence and possibility of video taking devices, such as mobiles and security cameras have inspired the search for video on face recognition which is very relevant in police applications. Whereas current paths have reported high accuracy errors, performance at lower prices for wrong acceptance requires important advancement. The choice of frames is followed by drawing features based on the representation of learning, where three hand-outs are represented 1) Deep learning architecture, which is a mixture of low stacking automatic encoder (SDAE) and deep machine Boltzmann (DBM) 2) formulation for joint illustration in an automatic encoder 3) Improve the DBM loss function, including low range modification. At last a multilayer neural network is used as a classifier to get the verification decision. The results are shown in two public databases on hand, YouTube Faces,Point and Shoot Challenge. The new study suggests that 1) frame selection based on the quality of the proposed features gets extraordinary and steady performance compared to the front frame, casual frames or plot selection using perceptual image quality dimensions without reference and 2) SDAE features of Common learning and low DBM and low regularization range helps get better facial confirmation.
\end{abstract}

Keywords: Deep Learning, Auto Encoder, Deep Boltzmann, Machine, Face Perception, Frame Selection

\section{INTRODUCTION}

VIDEO face identification has developed hugely important for security purposes. For instance, more than 80,000 people are recognized and verified in 2008 Beijing Olympics by face recognition in videos. With improvements in technology, video taking devices are available to a huge number of people in the form of small electronic devices like mobiles and tablets. In unrestrained scheme, videos captured by such devices may be used by law enforcement agency. So, there is a huge inspiration to use video data to do correct face acknowledgment. Fig. 1 shows frames from video clips in which the face parts have been cropped and detected. Though one frame from a video can only take partial matter, various frames capture a huge data about the face referring to its form under the effect of general covariates such as pose, and expression. By using the large range of information in a video, a strong and complete illustration of a face image can be extracted and precision can be enhanced.

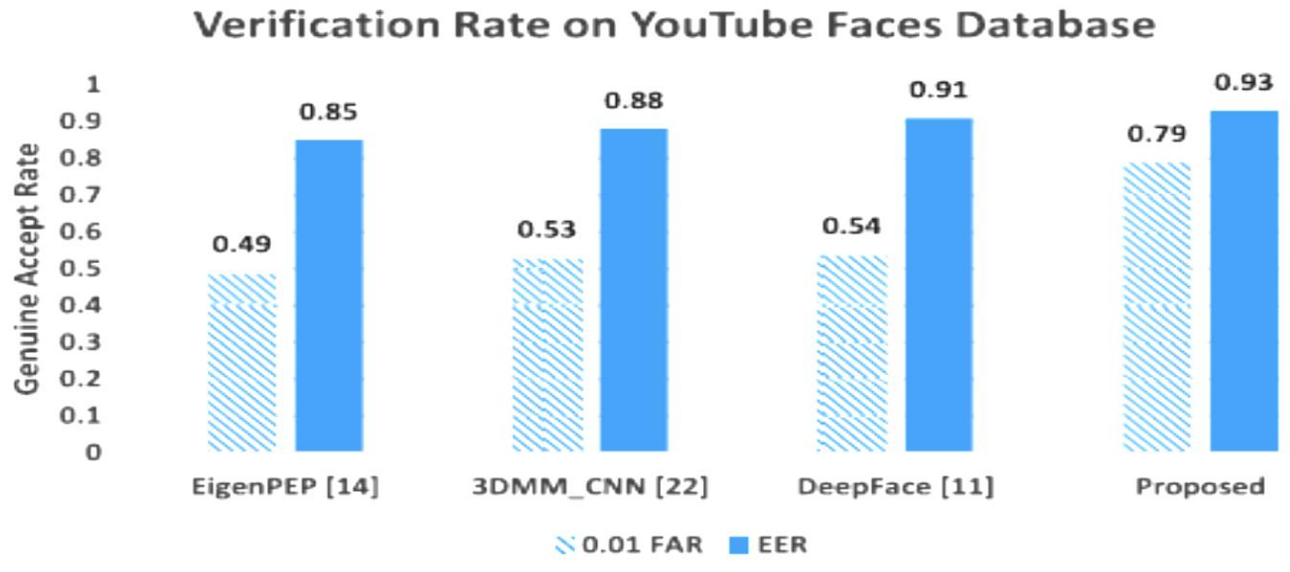

Fig1. Shortening the presentation of the best face confirmation algorithms on theYouTube faces files . 
It is clear that there is a high gap in the presentation at low error acceptance rates when compared to presentation at EER. We glass case that the projected algorithm does well even at a low error acceptance rate.

\subsection{Proposed system}

The proposed algorithm is separated into three parts: (i) frame choice (ii) deep learning based facet removal, and (iii) face confirmation by means of using above forms .

\subsection{Entropy Based Frame Choice}

Calculating the body pace and extent, a video clip of 4-6 seconds might also fit in one hundred-two hundred frames.Present copy for video face fame has both used all the frames, or handled a few chosen frames, or have implemented the algorithms for body option.Dealing out the frames can bring on addition of awful and unnecessary information. Proposed to detachment of the video into frame clusters and pick the maximum expert frames from each cluster using main issue analysis (PCA). Proposed to pick frames through analyzing pose and activity blur statistics for each frame by the use of energetic look fashions (AAM) and concluding on frames with controlled pose and minimum dullness. Jillela and Ross practical optical drift to make resolved frames by applying the usage of short 5 frame arrangements even as heading off the arrangements which confess extra interframe motion.

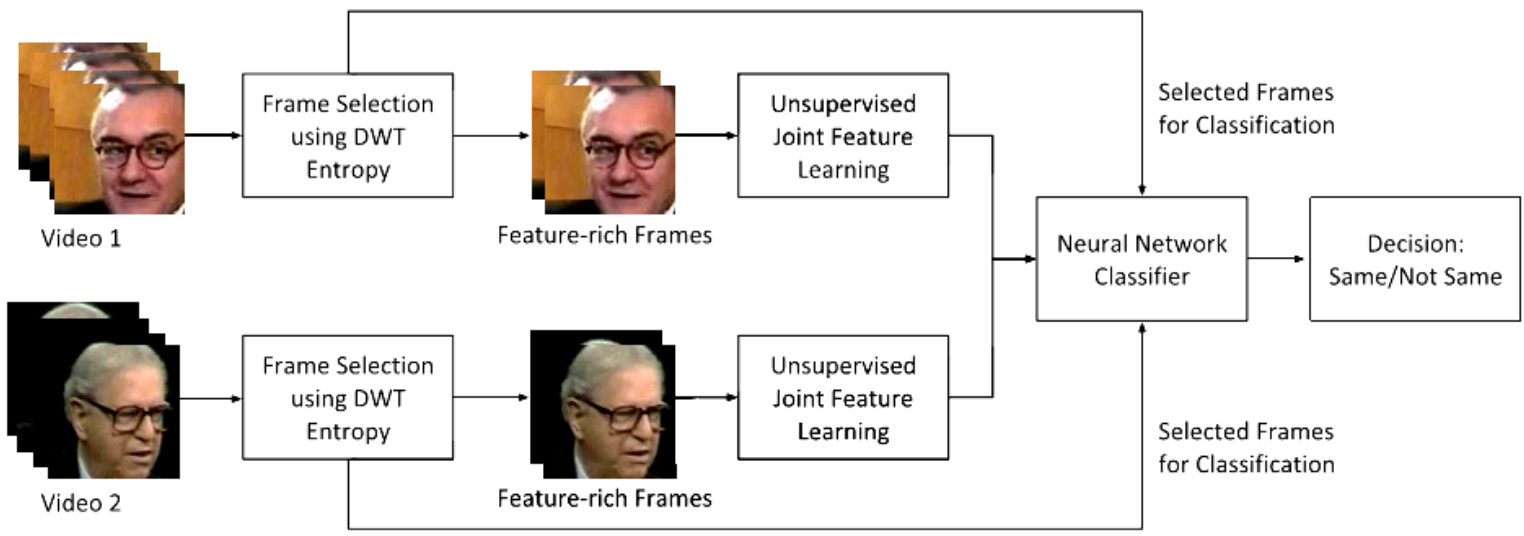

Fig2. Steps concerned in the projected face identification algorithm

The proposed procedure presents a remarkable attitude closer to body collection via utilizing quality richness as the principles. It is our declaration that quantifying the role richness of an image aid in derive the frames which have high hope of consist of biased effectiveness. A nice way to figure distinctive-richness, first the input (detected face) figure I is preprocessed to a smart size and distorted to grayscale through acting face identification first and allowing for near the facial place, we compose certain that the various non-face matter of the body does not interrupt with the projected algorithm.
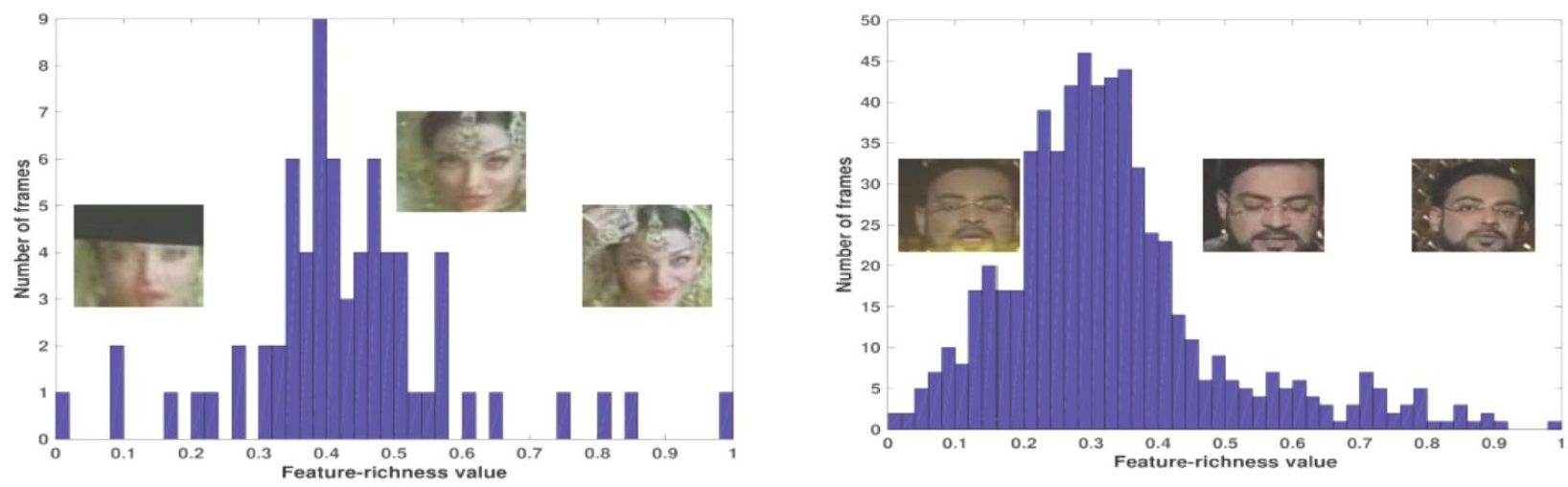

Fig. 3 Quality feature handling of two unlike videos. 
Some of the high quality feature (values near to 1) and least quality feature frames (values near to 0) are offered for design. We can observe that the reliability frames are authorize a high quality feature score and the low quality frames which display amount such as closure and smudge are authorized a low quality feature attain. Note down that the complete frames in the two videos are unlike.

Subsequently, the discrete wavelet transform(DWT) of the reprocessed image $i$ is caculated as follows:

$$
\left[\mathrm{i}_{\mathrm{Ap}}, \mathrm{i}_{\mathrm{Ho}}, \mathrm{i} \mathrm{Vr}, \mathrm{i}_{\mathrm{Dg}}\right]=\mathrm{DWT}
$$

$i A p$ takes the estimate coefficients of the picture, while $[i H o, i V r, i D g]$ contain the specify coefficients in horizontal, vertical, and diagonal sub-bands. The high and low pass filters used for disintegration depend on the sort of mother wavelet used. In this study, we used a bi-orthogonal mother wavelet which is balanced and capably encodes edge feature part. The aspect and estimate coefficients obtained using Eq. 1 signify the first level DWT coefficients. Another level of DWT is useful for the estimate band $i A p$ given below

$$
\left[\mathrm{i}_{A p}^{\prime}, \mathrm{i}^{\prime} \mathrm{Ho}_{\mathrm{o}}, \mathrm{i}^{\prime} \mathrm{Vr}, \mathrm{i}_{\mathrm{Dg}}^{\prime}\right]=\mathrm{DWT}\left(\mathrm{i}_{\mathrm{Ap}}\right)
$$

$i^{\prime} A p$ and [ $\left.i^{\prime} H o, i^{\prime} V r, i^{\prime} D g\right]$ signify the second step of DWT estimate and feature coefficients of input image $i$. DWT is helpful to allow multi-resolution study of the presented photo.Whereas the first step DWT includes the coefficients for the better facts of the picture, the second step DWT encrypt the total features whereas focusing less on well details. We have noticed that with photo of size $80 \times 100$ and below, the third step DWT is incapable to protect adequate edge matter and is not helpful for frame choice.As a result, in this study, we regard as only two steps of DWT.

\subsection{Deep Learning Based Facet Removal}

The moment in which high quality frames are seized, further includes function removal and toning.Various fields of the art of algorithms in present prose use convolution neural networks.In this study, we advise a stacked denoising autoencoders (SDAE) and Deep Boltzmann gadget (DBM) mainly based set of points that can adjourn proper output with guarded instruction data while at the same time being able to use the additional education info to advance improve total presentation. Foremost, we present an summary of SDAE and DBM guide by way of the presented structure.

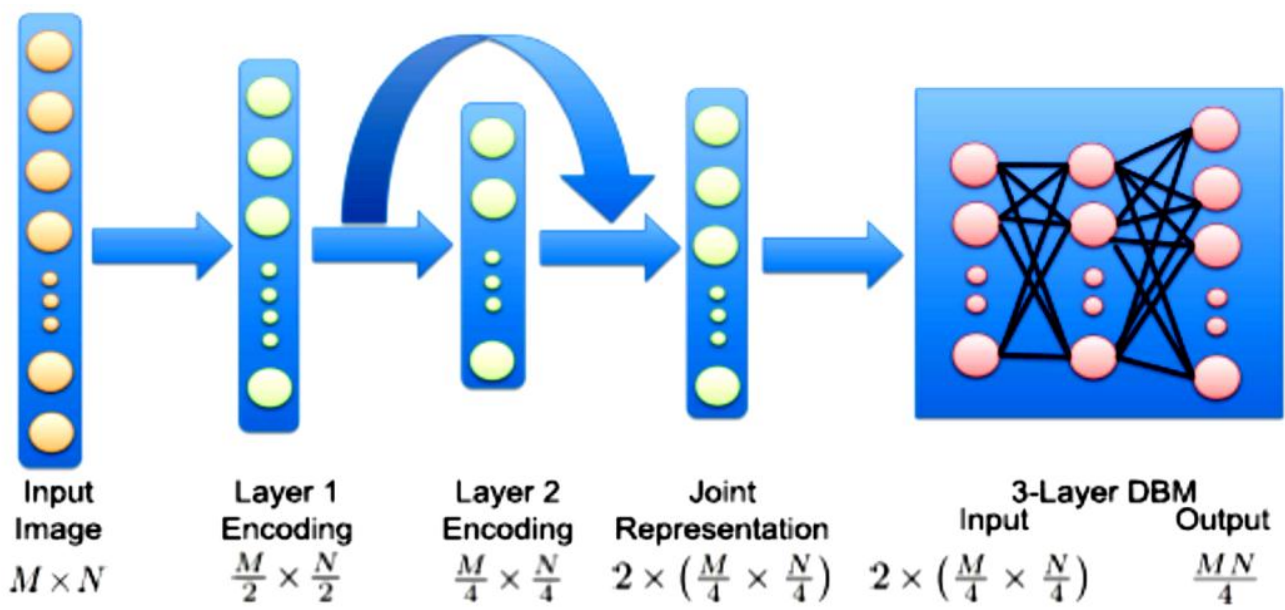

Fig. 4 Deep learning based facet removal

Commencing input layer, 2 unseen layer image are gauge using SDAE encoding function. A joint depiction is then found which unites the matter from two SDAE encoding layers. Using joint representation as input, a DBM is worn for gauge a final feature . 


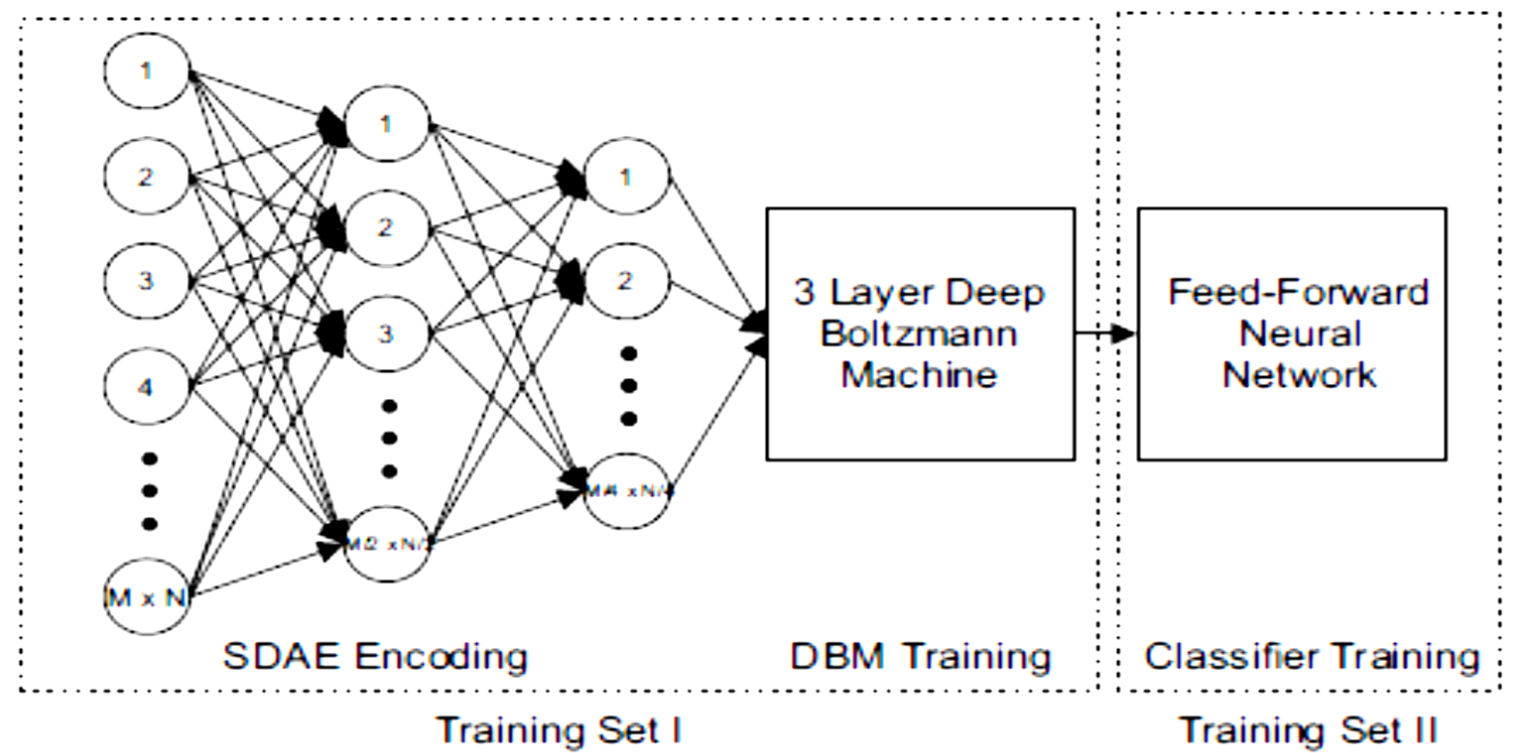

Fig 5. Deep learning structural design for face identification.

\section{METHODOLOGY}

The feature removal and toning are presented using a deep learning structural design that comprises of stacked denoising autoencoders (SDAE) and Deep Boltzmann Machine (DBM) for feature/image learning and neural network classification. Image 2 shows the steps include in the deep learning structural design. Foremost in brief we present an outline of SDAE and DBM pursued by the presented structural design.

\section{EXPERIMENTAL RESULTS}

Consecutively to estimate the efficiency of the presented design, face identification attempts are presented on two famous video standard databases: YouTube Faces and the Point and Shoot Challenge. Three unlike attempts are presented to exhibit the efficiency of the presented design.

- evaluate the presentation of state-of-the-art output described on these databases with the presented design.

- calculate the usefulness of individual works of the presented design and

- calculate the generality potential by checking the presentation with cross file training and testing pairs.

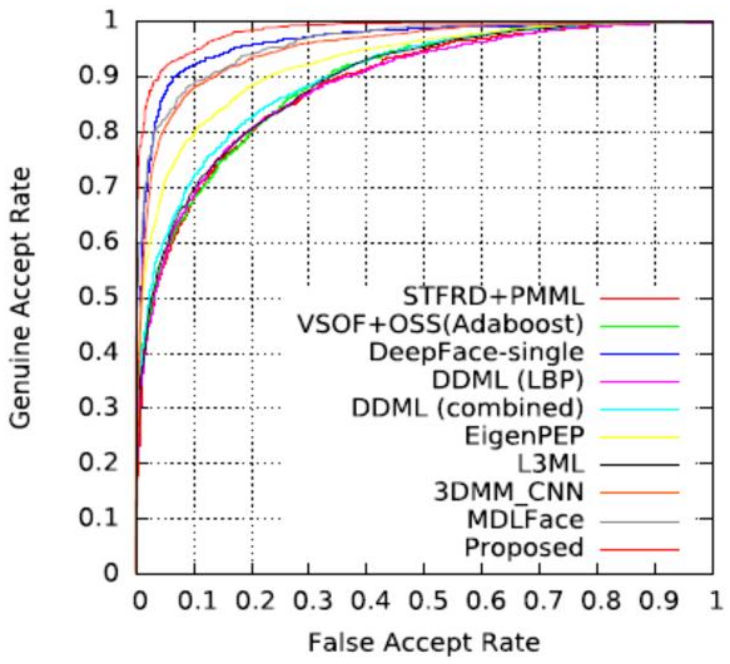

Fig-6: ROC curves analyzing the authentication presentation of the projected design with accessible outcome as noted on the YTF file home page. 


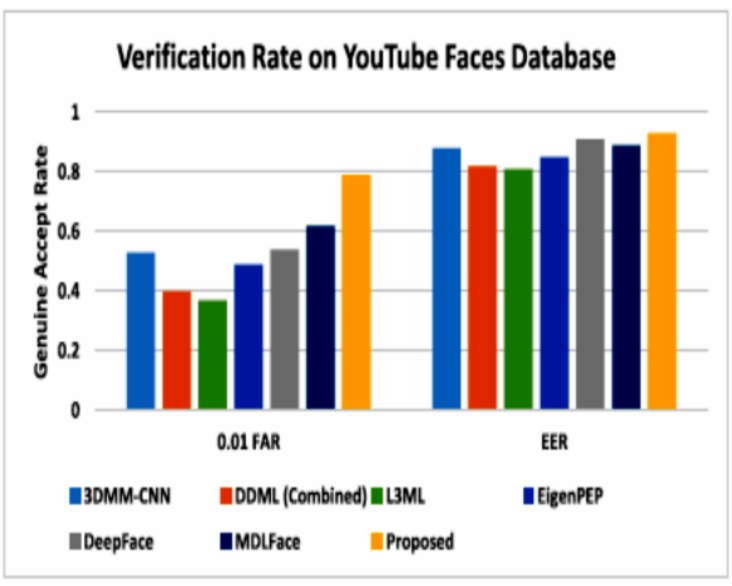

Fig. 7: Shortening the evidence of presentation of the projected design and state of the field designs on the youtube faces file.
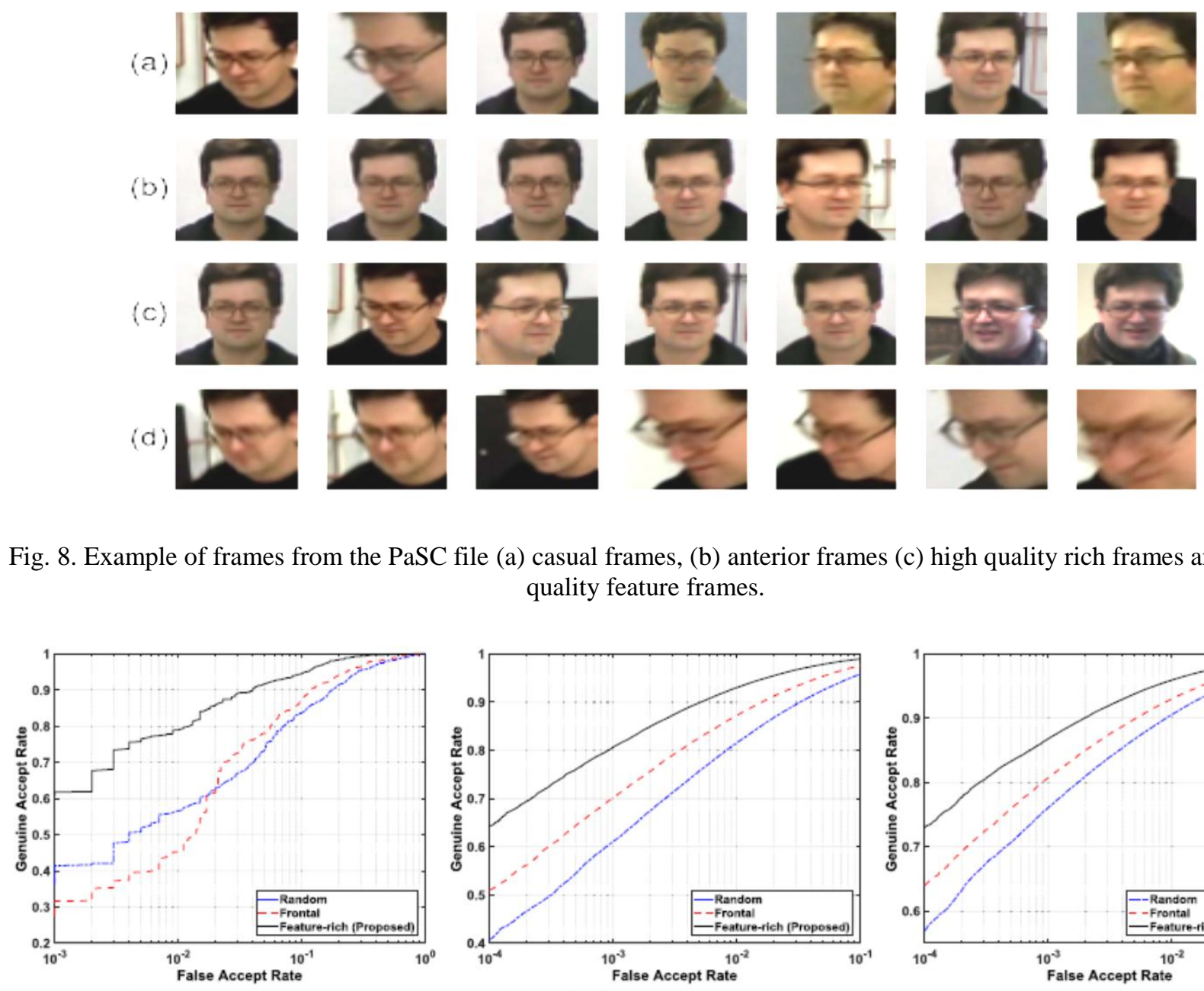

Fig. 8. Example of frames from the PaSC file (a) casual frames, (b) anterior frames (c) high quality rich frames and (d) low quality feature frames.

(a) YouTube Faces database

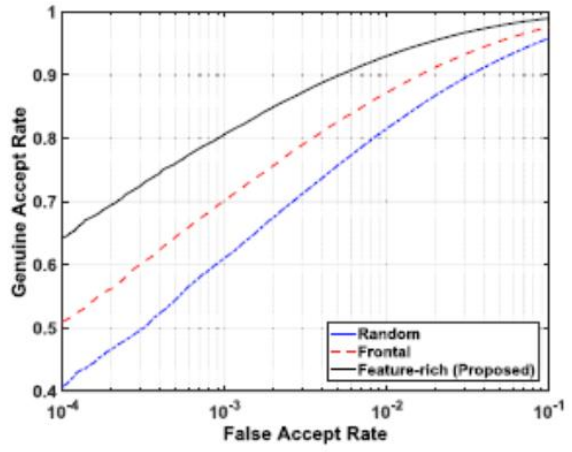

(b) Handheld subset of PaSC database

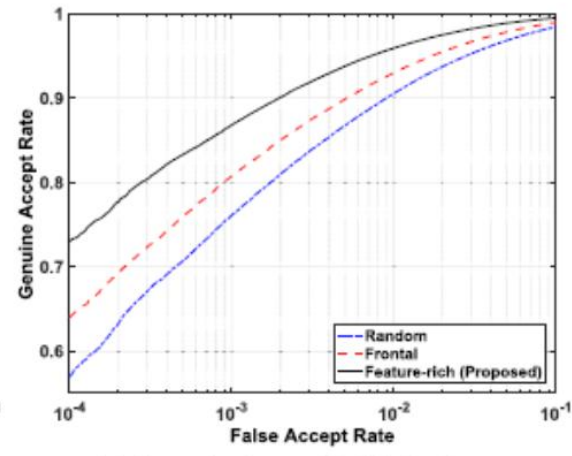

(c) Control subset of PaSC database

Fig.9. ROC curves analyzing the authentication presentation of the projected design with frame choice paths on the two files. (a) YouTube Faces files. (b) Handheld separation of PaSC file. (c) Manage separation of PaSC file. 


\section{CONCLUSION}

Confirming status in videos has numerous operations in social media, security and police force. Offered paths have attain high authentication certainity at equal false rate; though attaining high act at low error accept rate is static an tough investigate challenge. In this study, a original video face authentication design is projected which uses the frame choices and deep learning based facet removal.The projected design begins with attentively choosing high quality frames from input videos by means of wavelet disintegration and decline. The projected deep learning structural design which unites SDAE joint representation with DBM is worn to take out features from the choosed frames. The extracted image from two videos are coordinated using a feed forward neural network. The outputs are verified on the testing Point and Shoot Challenge and YouTube Faces files. The assessment with state-of-the-art outputs on both the files show that the projected design gives the best output on both of the files at low error accept rate, though with the limited training information. Apart from the standard code of both the files, various extra analysis have been projected to illustrate the usefulness of the projected assistance (i) joint feature learning in an auto encoder, (ii) sparse and low rank regularization in DBM, and (iii) grouping of SDAE and DBM in the projectec structural design. An upcoming study, we plan to widen the design for "face identification in crowd" with several subjects in every video.

\section{REFERENCES}

[1] Facial recognition technology safeguards Beijing Olympics, accessed on Mar. 10, 2017 [Online]. Available: http://english.cas.cn/ resources/archive/china_archive/cn2008/200909/t20090923_42959.shtml

[2] J. Beveridge et al., "The challenge of face recognition from digital point-and-shoot cameras," in Proc. IEEE Conf. Biometrics Theory, Appl. Syst., Oct. 2013, pp. $1-8$.

[3] L. Wolf, T. Hassner, and I. Maoz, "Face recognition in unconstrained videos with matched background similarity," in Proc. IEEE Conf. Comput. Vis. Pattern Recognit., Jun. 2011, pp. 529-534.

[4] L. Wolf and N. Levy, "The SVM-minus similarity score for video face recognition," in Proc. IEEE Conf. Comput. Vis. Pattern Recognit., Jun. 2013, pp. 3523-3530.

[5] H. Li, G. Hua, Z. Lin, J. Brandt, and J. Yang, "Probabilistic elastic matching for pose variant face verification," in Proc. IEEE Conf. Comput. Vis. Pattern Recognit., Jun. 2013, pp. 3499-3506.

[6] Z. Cui, W. Li, D. Xu, S. Shan, and X. Chen, "Fusing robust face region descriptors via multiple metric learning for face recognition in the wild," in Proc. IEEE Conf. Comput. Vis. Pattern Recognit., Jun. 2013

pp. 3554-3561.

[7] H. Méndez-Vázquez, Y. Martínez-Díaz, and Z. Chai, "Volume struc-tured ordinal features with background similarity measure for video face recognition," in Proc. Int. Conf. Biometrics (ICB), Jun. 2013,

pp. $1-6$.

[8] H. S. Bhatt, R. Singh, and M. Vatsa, "On recognizing faces in videos using clustering-based re-ranking and fusion," IEEE Trans. Inf. Forensics Security, vol. 9, no. 7, pp. 1056-1068, Jul. 2014.

[9] J. Y. Junlin Hu, J. Lu, and Y.-P. Tan, "Large margin multi-metric learning for face and kinship verification in the wild," in Proc. Asian Conf. Comput. Vis., 2014, pp. 252-267.

[10] J. Hu, J. Lu, and Y. Tan, "Discriminative deep metric learning for face verification in the wild," in Proc. IEEE Conf. Comput. Vis. Pattern Recognit., Jun. 2014, pp. 1875-1882.

[11] Y. Taigman, M. Yang, M. Ranzato, and L. Wolf, "DeepFace: Closing the gap to human-level performance in face verification," in Proc. IEEE Conf. Comput. Vis. Pattern Recognit., Jun. 2014, pp. 1701-1708.

[12] W. Wang, R. Wang, Z. Huang, S. Shan, and X. Chen, "Discriminant analysis on riemannian manifold of Gaussian distributions for face recognition with image sets," in Proc. IEEE Conf. Comput. Vis. Pattern Recognit., Jun. 2015, pp. 2048-2057.

[13] N. M. Khan, X. Nan, A. Quddus, E. Rosales, and L. Guan, "On video based face recognition through adaptive sparse dictionary," in Proc. IEEE Int. Conf. Workshops Autom. Face Gesture Recognit., May 2015, pp. 1-6.

[14] H. Li, G. Hua, X. Shen, Z. Lin, and J. Brandt, "Eigen-PEP for video face recognition," in Proc. Asian Conf. Comput. Vis., 2014, pp. 17-33.

[15] H. Li and G. Hua, "Hierarchical-PEP model for real-world face recog-nition," in Proc. IEEE Conf. Comput. Vis. Pattern Recognit., Jun. 2015, pp. $4055-4064$. 